\title{
Nonexistence of graphs with cyclic defect
}

\author{
Mirka Miller*† \\ School of Electrical Engineering and Comp. Science, University of Newcastle, Australia \\ Department of Mathematics, University of West Bohemia, Czech Republic \\ Department of Computer Science, King's College London, UK
}

Submitted: Nov 10, 2010; Accepted: Mar 9, 2011; Published: Mar 31, 2011

Mathematics Subject Classification: 05C35, 05C75

\begin{abstract}
In this note we consider graphs of maximum degree $\Delta$, diameter $D$ and order $\mathrm{M}(\Delta, D)-2$, where $\mathrm{M}(\Delta, D)$ is the Moore bound, that is, graphs of defect 2 . In 1] Delorme and Pineda-Villavicencio conjectured that such graphs do not exist for $D \geq 3$ if they have the so called 'cyclic defect'. Here we prove that this conjecture holds.
\end{abstract}

Keywords: Graphs with cyclic defect, Moore bound, defect, repeat.

\section{Nonexistence of graphs with cyclic defect}

Let $G$ be a graph of maximum degree $\Delta$, diameter $D$ and order $\mathrm{M}(\Delta, D)-2$, where $\mathrm{M}(\Delta, D)=1+\Delta+\Delta(\Delta-1)+\Delta(\Delta-1)^{2}+\cdots+\Delta(\Delta-1)^{D-1}$ is the Moore bound, that is, graphs of defect 2 . In such a graph $G$ any vertex $v$ can reach within $D$ steps either two vertices (called repeats of $v$ ) in two different ways each, or one vertex (called double repeat of $v$ ) in three different ways; all the other vertices of $G$ are reached from $v$ in at most $D$ steps in exactly one way. The repeat (multi)graph of $G, R(G)$, consists of the vertex set $V(G)$ and there is an edge $\{u, v\}$ in $R(G)$ if and only if $v$ is a repeat of $u$ (and vice versa) in $G$. Clearly, when defect is $2, R(G)$ is either one cycle of length $n=|V(G)|$ or a disjoint union of cycles whose sum of lengths is equal to $n$. If $R(G)$ is cycle of length $n$ then we say that $G$ has cyclic defect. Interest in such graphs is part of the general study of the

\footnotetext{
*mirka.miller@newcastle.edu.au

${ }^{\dagger}$ This research was supported by a Marie Curie International Incoming Fellowship within the 7th European Community Framework Programme
} 
degree/diameter problem. For a survey of this problem, see [4]. Graphs with cyclic defect were first studied by Fajtlowicz [2] who proved that when $D=2$ the only graph with cyclic defect is the Mobius ladder on 8 vertices (with $\Delta=3$ ). Subsequently, for $D \geq 3$, Delorme and Pineda-Villavicencio [1] proposed several ingenious algebraic techniques for dealing with graphs with cyclic defect and they proved the nonexistence of such graphs for many values of $D$ and $\Delta$. They conjectured that graphs with cyclic defect do not exist for $D \geq 3$. In this paper we use structural properties of graphs with cyclic defect to prove that this conjecture holds.

Observation 1.1 For $\delta<1+(\Delta-1)+(\Delta-1)^{2}+\ldots+(\Delta-1)^{D-1}, \Delta \geq 3$ and $D \geq 2$, a graph of defect $\delta$ must be regular.

It is also easy to see that there are no graphs with cyclic defect of degree $\Delta=2$. Therefore, from now on we assume $G$ to be a $\Delta$-regular graph with cyclic defect, degree $\Delta \geq 3$, and diameter $D \geq 3$.

We say that $S \subset V(G)$ is a closed set of repeats if for every vertex of $S$ none of its repeats is outside of $S$. Clearly, a graph with cyclic defect cannot contain a closed set of repeats that is of cardinality less that $|V(G)|$.

We denote by $\Theta_{D}$ the union of three independent paths of length $D$ with common endvertices. Since the $3 D-1$ vertices of $\Theta_{D}$ comprise a closed set of repeats, while $G$ contains $\Delta\left(1+(\Delta-1)+(\Delta-1)^{2}+\cdots+(\Delta-1)^{D-1}\right)-1$ vertices, we have

Observation 1.2 Graph with cyclic defect does not contain $\Theta_{D}$.

Suppose $G$ contains a cycle $C$ of length $2 D-m, m>1$. Then for every vertex $v$ on $C$, there are more than 2 vertices on $C$ that are repeats of $v$. Since each vertex has at most two distinct repeats, we have immediately that $m \leq 1$. Moreover, if $m=1$ then $C$ is a closed set of repeats consisting of $2 D-1$ vertices, while $G$ contains $\Delta(1+(\Delta-1)+(\Delta-$ $\left.1)^{2}+\cdots+(\Delta-1)^{D-1}\right)-1$ vertices, a contradiction for every $\Delta \geq 3$. Therefore, we have

Observation 1.3 Graph with cyclic defect does not contain a cycle of length less than $2 D$.

This means that the girth of $G$ is $2 D$, and every vertex $v$ is contained in exactly two $2 D$-cycles, and no other cycle of length at most $2 D$.

Let $S$ be a set of vertices in $G$ and $H$ a subgraph of $G$. We denote by $S^{\prime}=\operatorname{rep}^{H}(S)$ the set of repeats of $S$ that occur in $H$. Furthermore, two $2 D$-cycles $C^{1}$ and $C^{2}$ are called neiqhbouring cycles if they have non-empty intersection. The following lemma was proved in [3] ; it will be used to prove the main result of this paper. 
Lemma 1.1 (Repeat Cycle Lemma) [S] Let $G$ be a graph with $D \geq 4$ and $D \geq 2$, and defect 2. Let $C$ be a $2 D$-cycle in $G$. Let $\left\{C^{1}, C^{2}, \ldots, C^{k}\right\}$ be the set of neighbouring cycles of $C$, and $I_{i}=C^{i} \cap C$ for $1 \leq i \leq k$. Suppose at least one $I_{j}$, for $j \in\{1, \ldots, k\}$, is a path of length smaller than $D-1$. Then, there is an additional $2 D$-cycle $C^{\prime}$ in $G$, called repeat cycle, intersecting $C^{i}$ at $I_{i}^{\prime}=\operatorname{rep}^{C^{i}}\left(I_{i}\right)$, where $1 \leq i \leq k$.

For an illustration, see Fig. [1]

Corollary 1.1 If $C$ and $C^{\prime}$ are repeat cycles of each other then they comprise a closed set of $4 D$ repeats.

Proof. Consider an arbitrary vertex $x \in C \cap I_{i}, i \in 1, \ldots, k$. The vertex $x$ has two repeats: one of them is the vertex on $C$ that is at distance $D$ from $x$. The second repeat of $x$ is on the intersection of the repeat cycle $C^{\prime}$ and $I_{i}^{\prime}$. Since $C$ and $C^{\prime}$ are repeat cycles of each other, we have $R(C)=C \cup C^{\prime}=R\left(C^{\prime}\right)$ and so $C \cup C^{\prime}$ is a closed set of repeats.

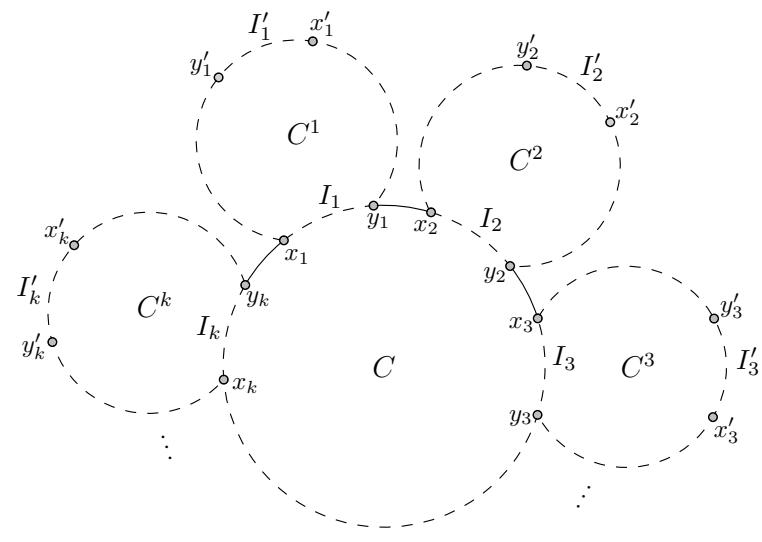

(a)

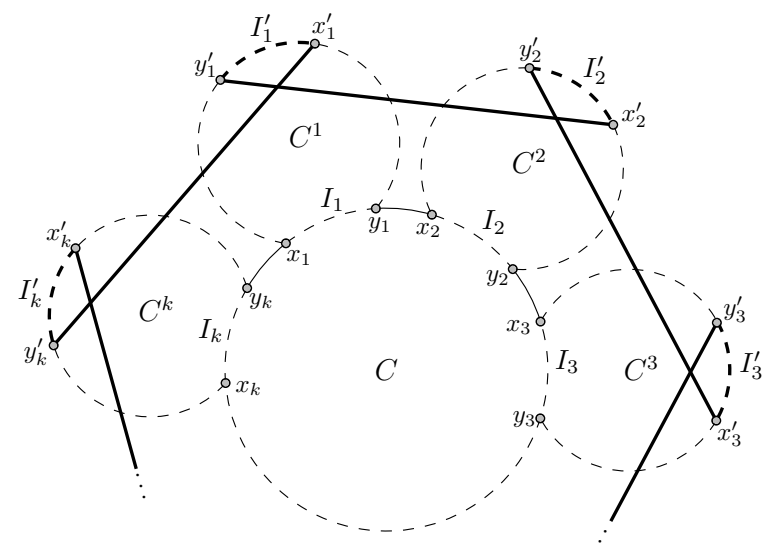

(b)

Figure 1: Illustration for Lemma [1.1] [3].

We are now ready to prove the main result.

Theorem 1.1 Graphs with cyclic defect do not exist for $\Delta \geq 3$ and $D \geq 3$.

Proof. Let $G$ be a graph with cyclic defect. Let $C$ be a cycle of length $2 D$ in $G$. We need to consider two cases.

Case 1. There exist two $2 D$-cycles, say $C_{1}$ and $C_{2}$, with intersection that is a path of length smaller than $D-1$. Then, by Corollary 1.1. cycle $C_{1}$ has a repeat cycle $C_{1}^{\prime}$ and the two cycles $C_{1}$ and $C_{1}^{\prime}$ comprise a closed set of $4 D$ repeats, a contradiction since $G$ is a graph with cyclic defect and $\Delta\left(1+(\Delta-1)+(\Delta-1)^{2}+\cdots+(\Delta-1)^{D-1}\right)-1$ vertices. 


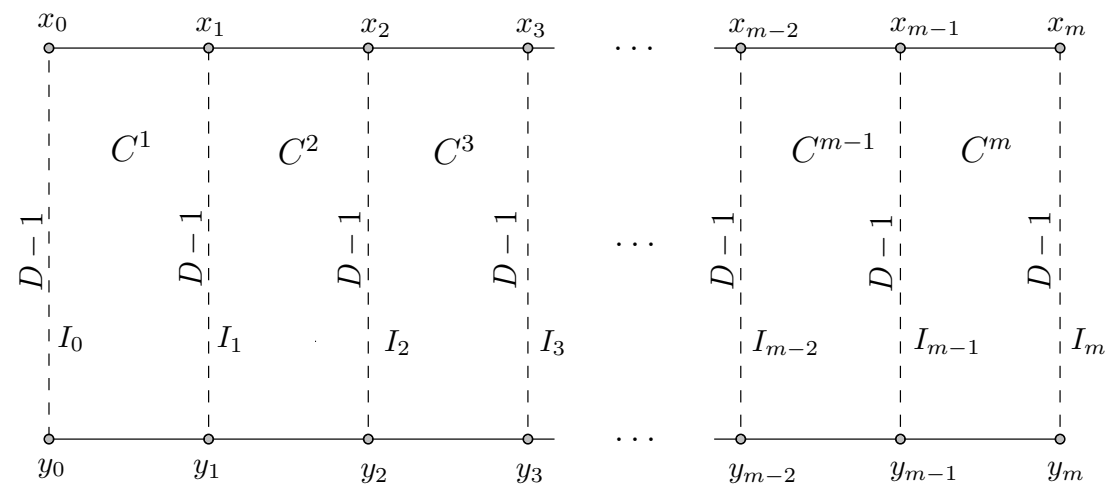

(a)

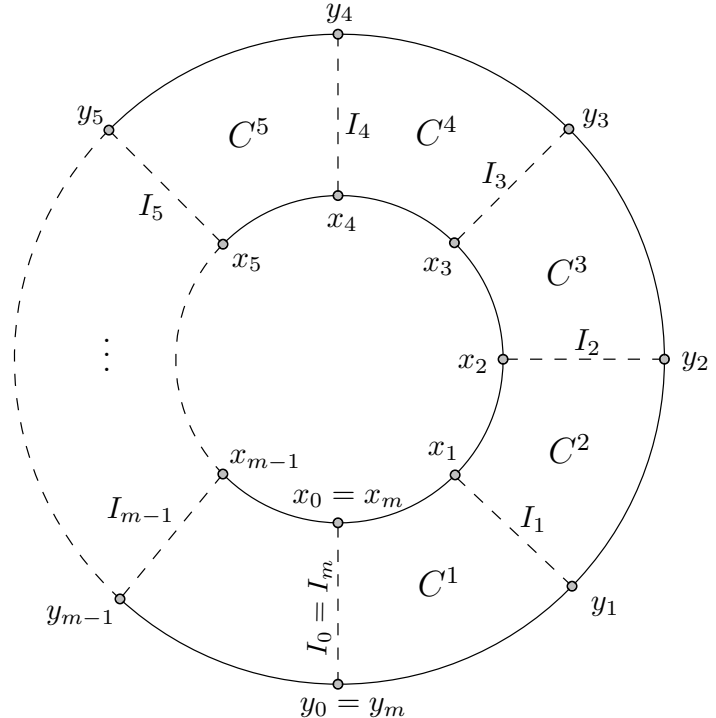

$(b)$

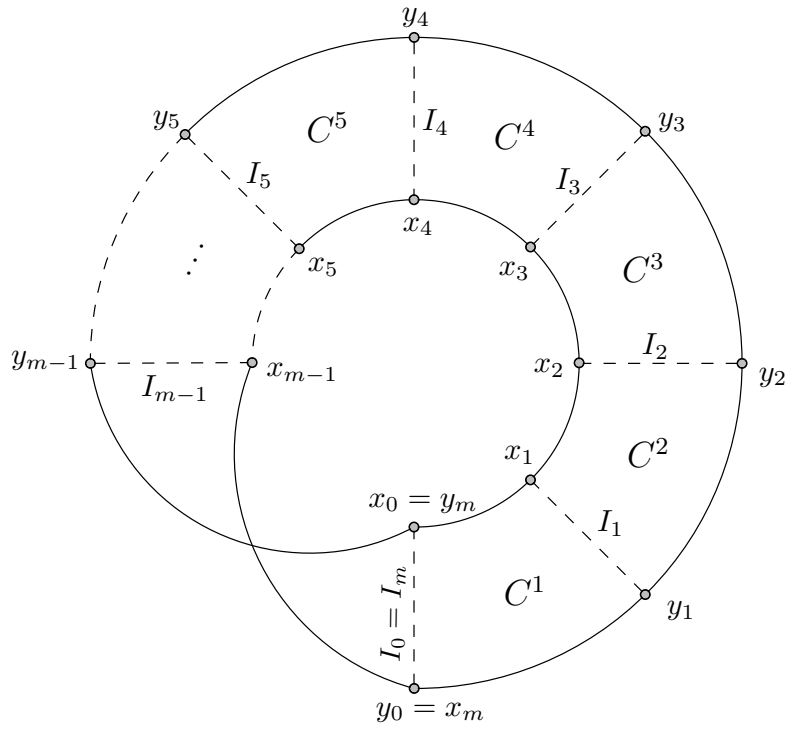

$(c)$

Figure 2: Illustration for Case 2 of the proof of Theorem $1.1[3]$.

Case 2. There do not exist two cycles with intersection that is a path of length smaller than $D-1$. That is, any two $2 D$-cycles have either empty intersection or they intersect in a path of length exactly $D-1$. Recall that the length of the path cannot be more since there are no $\Theta_{D}$. Then $G$ contains as a subgraph a succession of $2 D$-cycles $C_{m}, C_{1}$, $C_{2}, \ldots, C_{m-1}$ such that any two consecutive cycles have intersection a path of length $D-1$ (that is, they share $D$ vertices). Assume that the value of $m$ is maximum possible. Refer to Fig 2(a). Since $G$ is finite, $C_{1}$ and $C_{m}$ must also intersect in a path of length $D-1$. 
There are two possibilities, depicted in Fig. 2(b) and (c). Clearly, in the first case the vertices $x_{1}, x_{2}, \ldots, x_{m}$ form a closed set of repeats for any $\Delta \geq 3$, and this set does not include the vertices $y_{1}, y_{2}, \ldots, y_{m}$ so that $G$ does not have cyclic defect.

In the second case, for any $\Delta \geq 3$, the vertices $x_{1}, x_{2}, \ldots, x_{m}$ and the vertices $y_{1}, y_{2}, \ldots, y_{m}$ together form a closed set of repeats consisting of $2 \mathrm{~m}$ vertices which however does not include all the vertices of $G$ if $D \geq 3$, a contradiction.

\section{References}

[1] C. Delorme and G. Pineda-Villavicencio, On graphs with cyclic defect, Electron. J. Combin. 17 (2010), \#R143.

[2] S. Fajtlowicz, Graphs of diameter 2 with cyclic defect, Colloquium Mathematicum 51 (1987), 103-106.

[3] R. Feria-Purón, M. Miller and G. Pineda-Villavicencio, On graphs of defect at most 2, preprint (2010).

[4] M. Miller and J. Širáň, Moore graphs and beyond: A survey of the degree/diameter problem, Electronic J. Combin. 11 (2005), \#DS14. 